\title{
CAN COMPOSITE FLUFFY DUST PARTICLES SOLVE THE INTERSTELLAR CARBON CRISIS?
}

\author{
Eli Dwek ${ }^{1}$
}

\begin{abstract}
Recent observations suggest that the abundance of carbon in the interstellar medium is only $\sim 60 \%$ of its solar value, and that other heavy elements may be depleted by a similar amount. Furthermore, more than half of the interstellar carbon is observed to be in the gas in the form of $\mathrm{C}^{+}$, leaving less than $\sim 40 \%$ of the solar carbon abundance available for the dust phase. These observations have created the so-caled interstellar "carbon crisis", since traditional interstellar dust models require about twice that value to be tied up in graphite grains in order to explain the interstellar extinction curve.

Recently, Mathis (1996) suggested a possible solution to this crisis. In his newly-proposed dust model the majority of the interstellar carbon is contained in composite and fluffy grains that are made up of silicates and amorphous carbon grains, with $45 \%$ of their volume consisting of vacuum. Per unit mass, these grains produce more UV extinction, and can therefore account for the interstellar extinction curve with about half the carbon required in traditional dust models.

This paper presents an detailed assessment of the newly-proposed dust model, and concludes that it falls short in solving the carbon crisis, in providing a fit to the UV-optical interstellar extinction curve. It also predicts a far-infrared emissivity in excess of that observed with the COBE/DIRBE and FIRAS instruments from the diffuse interstellar medium. This excess infrared emission is a direct consequence of the lower albedo of the composite fluffy dust particles, compared to that of the traditional MRN mixture of bare silicate and graphite grains. The failure of the new model highlights the interrelationships between the various dust properties and their observational consequences, and the need to satisfy them all simultaneously in any comprehensive interstellar dust model.

In light of these problems, the paper examines other possible solutions to the carbon crisis.
\end{abstract}

Subject headings: interstellar: grains, abundances infrared: radiation - spectrum, ISM: dust, extinction

\footnotetext{
${ }^{1}$ Laboratory for Astronomy and Solar Physics, Code 685, NASA/Goddard Space Flight Center, Greenbelt, MD 20771. e-mail address: eli.dwek@gsfc.nasa.gov
} 


\section{INTRODUCTION}

The most commonly used interstellar dust model, first introduced by Mathis, Rumpl \& Nordsieck (1977; hereafter MRN), consists of two distinct populations of bare silicate and graphite particles with an $a^{-3.5}$ power law distribution in grain sizes extending approximately from 0.0050 $\mu \mathrm{m}$ to $0.25 \mu \mathrm{m}$. With the optical constants of Draine \& Lee (1984) and Draine (1985) the model is very succesful in reproducing the average intestellar extinction curve, the 9.7 and $18 \mu \mathrm{m}$ silicate extinction features, and the average interstellar albedo and polarization. The model requires that about $75 \%$ of the solar abundance of carbon be locked up in graphite grains, and essentially all the solar abundance of $\mathrm{Mg}, \mathrm{Si}$, and $\mathrm{Fe}$ and about $12 \%$ of the solar abundance of oxygen be locked up silicate dust (assuming a silicate composition of $\left\{\mathrm{MgSiFe} \mathrm{O}_{4}\right.$ ).

There have recently been various lines of evidence for the case that the abundances of various heavy elements in the interstellar medium (ISM) gas are less than their nominal solar values. Concentrating on carbon, its solar abundance, normalized to a hydrogen abundance of $10^{6} \mathrm{H}$-atoms, is $Z_{C, \odot} \approx 355$ (Grevesse, Noels, \& Sauval 1996). In the ISM, the abundance of this element is significantly lower, only $225 \pm 50 \mathrm{C}$-atoms (Snow \& Witt 1995). This value is based on observations of young stars whose photospheric carbon abundances reflect those of the interstellar medium (gas plus dust phases) at the time of their formation. A significant amount of this interstellar carbon abundance is in the gas phase. Using the Goddard High Resolution Spectrograph on board the Hubble Space Telescope, Cardelli et al. (1996) inferred a C abundance of $140 \pm 20$ atoms from C II] $\lambda 2325 \AA$ absorption measurements in the direction of six stars. This leaves a total of $85 \pm 55 \mathrm{C}$-atoms available for the dust phase, compared to the $\sim 300$ atoms required by the MRN and various other dust models to be in solids. This has created what Kim \& Martin (1996) termed the " $\mathrm{C} / \mathrm{H}$ crisis". Furthermore, there is evidence that the ISM abundances of other heavy element, such as nitrogen and oxygen, are depleted by as much as $60-70 \%$ compared to their solar value (see review by Mathis 1996). The crisis is therefore not confined to carbon alone, since dust models use up essentially all the available solar abundance of refractory elements such as $\mathrm{Mg}, \mathrm{Si}, \mathrm{Ca}, \mathrm{Ti}$, and Fe.

This carbon crisis has motivated Mathis (1996) to propose a new interstellar dust model in which most of the dust is bonded together in the form of a loose aggregate of dust grains. A previous version of this model, and the motivations behind it were presented by Mathis \& Whiffen (1989). Simply put, these fluffy aggregates can produce more extinction per unit mass than their combined individual constituent dust grains. An interstellar dust model with fluffy aggregates as a major dust component will therefore require a minimal amount of carbon and silicate dust, and perhaps be consistent with current interstellar abundances constraints.

In addition to the abundance constraints, any interstellar dust model should be able to explain the observed interstellar extinction, albedo, polarization, and infrared (IR) emission. In this paper we concentrate on the abundance, extinction, albedo, and IR emission constraints placed on the composite fluffy dust (CFD) model presented by Mathis. The detection of $3.5-1000 \mu \mathrm{m}$ emission 
from high lattitude cirrus clouds by the Diffuse Infrared Background Experiment (DIRBE) and the Far Infrared Absolute Spectrophotometer (FIRAS) instruments on board the COBE satellite (Bernard et al. 1994; Weiland et al. 1996; Arendt et al. 1997, Dwek et al. 1997) provide strong constraints on interstellar dust models. The emission in the $3.5-12 \mu \mathrm{m}$ regime can be used to derive the abundance of the carriers of the $3.3,6.2,7.7,8.6$, and $11.3 \mu \mathrm{m}$ features, commonly referred to as the unidentified infrared emission bands (UIBs). The amount of carbon in these carriers has to be added to any interstellar dust model. The $C O B E$ observations also provide an estimate of the fraction of the total IR radiation that is emitted by these particles. They must therefore be responsible for a significant fraction of the integrated UV-optical extinction. Finally, changes in the UV-optical properties of the dust can alter their IR emissivities and temperatures, effects that can be detected in the observed spectrum of the diffuse ISM.

\section{THE OPTICAL PROPERTIES OF COMPOSITE FLUFFY DUST (CFD) PARTICLES}

The new Mathis model consists of very small graphite grains which are required to produce the $2200 \AA$ extinction feature, very small silicates, and composite particles consisting of loosely packed amorphous carbon and silicates particles. In order to minimize the amount of carbon needed to be in the solid phase, the model uses only $55 \mathrm{C}$-atoms in the form of graphite, just enough to produce the $2200 \AA$ bump. The remaining model parameters are then varied in order to produce the best fit to the interstellar extinction curve of Cardelli, Clayton, \& Mathis (1989) with the minimum amount of carbon and silicate dust. For amorphous carbon, Mathis found that the samples of type Be (see Rouleau \& Martin 1991) provided the best fit to the extinction data. The silicate dust is assumed to be composed of $\left\{\mathrm{MgSiFe} \mathrm{O}_{4}\right.$ with $\mathrm{Ca}, \mathrm{Al}$, and Ti oxides as additional minor constitutents. We characterize the silicate abundance by the number of silicate atoms locked up in the dust, with the understanding that a comparable number of $\mathrm{Mg}$ - and Fe-atoms, and about 4 times as many O- atoms are locked up in the dust as well.

We reproduced the Mathis CFD model with dust particles with the following characteristics (abundances quoted are normalized to $10^{6} \mathrm{H}$-atoms): (1) graphite grains with a $\mathrm{C}$ abundance of 55 ; (2) silicate particles with a $\mathrm{Si}$ abundance of 6.5 ; and (3) composite particles containing $105 \mathrm{C}$-atoms in amorphous form, $26 \mathrm{Si}$-atoms in silicates, and vacuum. The total dust phase abundance of silicate is thus 32.5 , about $85-92 \%$ of its solar abundance of $\sim 35.5-38$. The fractional volume occupied by these three composite grain constituents are 17, 38, and 45\%, respectively. The mass density of the three grain constituents are $2.25 \mathrm{~g} / \mathrm{cm}^{3}$ for the graphite particles; $3.3 \mathrm{~g}$ $\mathrm{cm}^{-3}$ for the silicates; and, adopting a value of $1.85 \mathrm{~g} \mathrm{~cm}^{-3}$ for the amorphous carbon, $1.55 \mathrm{~g}$

$\mathrm{cm}^{-3}$ for the composite fluffy particles. Interstellar grains are not spherical. Consequently, we followed Mathis' procedure, and multiplied the extinction calculated for the spherical bare silicates and the composite particles by a factor of 1.09, to account for the increased extinction due to the oblateness of the dust particles. 
The dielectric properties of composite particles can be derived by averaging those of their individual dust constituents. Two of the most common rules used to derive the dielectric properties of inhomogeneous particles are the Maxwell-Garnett, and the Bruggemann rule (see Bohren \& Huffman 1983). The former rule regards the composite particle as a matrix with embedded inclusions, and is not invariant under the interchange of matrix and inclusions. The Bruggeman rule is, and for a composite particle consisting of three constituents (amorphous carbon, silicates, and vacuum), the average dielectric constant $\epsilon_{a v}$ is given by the solution of a cubic equation (see Bohren \& Huffman eq. [8.51]):

$$
\sum_{j=1}^{3} f_{j} \frac{\epsilon_{j}-\epsilon_{a v}}{\epsilon_{j}+2 \epsilon_{a v}}
$$

where $\epsilon_{j}$ is the dielectric constant of the $\mathrm{j}^{\text {th }}$ constituent, and where the sum of the volume filling factors, $f_{j}$, of the constituent material is unity. Figure 1 compares the dielectric constants of the composite particle with those of its constituents as a function of wavelength. The dielectric constants of the silicate grains were taken from Draine \& Lee (1984), and those for amorphous carbon from Rouleau \& Martin (1991).

The size distribution of the composite fluffy particles is given by (Mathis 1996):

$$
f(a)=a^{-\alpha_{0}} \exp \left[-\left(\alpha_{1} a+\alpha_{2} / a+\alpha_{3} a^{2}\right)\right]
$$

where $a$ is the grain radius, and the parameters $\left\{\alpha_{0}, \alpha_{1}, \alpha_{2}, \alpha_{3}\right\}$ are equal to $\{3.5,0.0033,0.437$, $50\}$, respectively (Mathis 1996, private communication). The size distribution is concentrated around $0.1 \mu \mathrm{m}$, dropping to $10 \%$ of its peak value at $a \approx 0.05$, and $0.2 \mu \mathrm{m}$.

Two factors contribute to the enhancement in the extinction of the composite grains at infrared wavelength. This can be seen from the extinction optical depth at wavelength $\lambda$ per H-column density which is given by:

$$
\begin{aligned}
\tau_{\text {ext }}(\lambda) / N_{H} & =\mathcal{M}_{d}<\kappa_{d}(\lambda)> \\
& =\left(\mathcal{M}_{d} / \rho\right)<3 Q_{\text {ext }} / 4 a>
\end{aligned}
$$

where $\mathcal{M}_{d}$ is the dust mass column density, and $\left\langle\kappa_{d}(\lambda)>\equiv<3 Q_{\text {ext }} / 4 \rho a>\right.$ is the size-averaged dust mass extinction coefficient at wavelength $\lambda$, where $Q_{e x t}$ is the extinction efficiency of a grain of radius $a$ and mass density $\rho$. The mass column density of the composite grains is equal to the sum of the mass column densities of its grain constituents. However, because of its porosity its mass density, $\rho$, is smaller than theirs. As a result, the extinction of the composite grains is larger than the sum of the extinction of its consituent particles, which is the main reason for the enhanced extinction at UV-optical wavelengths. At long wavelengths $(\lambda \gtrsim 1 \mu \mathrm{m})$, an additional factor plays a role in increasing the extinction of the composite particles, namely an increase in their value of $\mathrm{Q}_{\text {ext }}$ compared to that of the amorphous carbon and silicate grains. In the Rayleigh limit, when $2 \pi a / \lambda \ll 1, \mathrm{Q}_{\text {ext }} / a$ can be written in terms of $\epsilon_{1}$, and $\epsilon_{2}$, the real and imaginary parts 
of the dielectric constant as:

$$
Q_{e x t} / a=\frac{24 \pi}{\lambda} \frac{\epsilon_{2}}{\left(\epsilon_{1}+2\right)^{2}+\epsilon_{2}^{2}}
$$

The value of $\mathrm{Q}_{\text {ext }} / a \rightarrow 0$ when either $\epsilon_{2} \ll \epsilon_{1}$, or $\epsilon_{2} \gg 1$. It has a maximum when $\epsilon_{2} \approx \epsilon_{1}+2$. Figure 1 shows that at long wavelengths $(\lambda \gtrsim 100 \mu \mathrm{m}), \epsilon_{2} \ll \epsilon_{1}$ for silicates, and $\epsilon_{2} \gg 10$ for amorphous carbon. For the composite grains $\epsilon_{2} \approx \epsilon_{1}+2 \approx 10$, yielding a larger value of $Q_{\text {ext }} / \mathrm{a}$ over that of amorphous carbon or silicate grains. So at far-IR wavelengths, the averaging of the optical properties plays an important role in increasing the absorptivity/emissivity of the composite grains. As a result, the composite fluffy grains are somewhat cooler than either graphite or silicate grains of identical radius.

\section{THE EFFECTS OF THE INCLUSION OF PAHs ON THE COMPOSITE FLUFFY DUST MODEL}

\subsection{The Infrared Emission}

An important test for the validity of the CFD model is if it can reproduce the observed $C O B E / D I R B E$ and FIRAS 3.5 - $1000 \mu \mathrm{m}$ emission from the diffuse ISM (Bernard et al. 1994, Weiland, Arendt, \& Sodroski 1996, Arendt et al. 1997, Dwek et al. 1997). The Mathis model does not include carriers of the UIBs which are necessary to account for the emission in the 3.5, 4.9, and $12 \mu \mathrm{m}$ DIRBE bands (Dwek et al. 1997). So a priori, the CFD model needs to be ammended to include UIB carriers. For calculational purposes we identified these carriers with polycyclic aromatic hydrocarbons (PAHs), with extinctions and IR properties as given by Désert et al. (1990). With this PAH model, most of the $12 \mu \mathrm{m}$ and all shorter wavelength diffuse emission is produced by these particles. The abundance of PAHs can therefore be directly determined from the near-IR COBE observations of the diffuse ISM (see Dwek et al. 1997 for details of the model). The PAH abundance required to reproduce the $C O B E$ data was added to the CFD model.

We note here that whereas the diffuse ISM spectrum obtained by the Infrared Astronomical Satellite (IRAS) could be reproduced by a simple extension of the graphite or silicate grain size distribution to very small sizes (Draine \& Anderson 1985; Weiland et al. 1986), the DIRBE spectrum cannot be fitted by the same method. The DIRBE extends the IRAS 12, 25, 60, and $100 \mu \mathrm{m}$ observations to shorter (and longer) wavelengths, and the near-IR 3.5 and 4.5 $\mu \mathrm{m}$ colors observed by DIRBE from the diffuse ISM are inconsistent with those calculated for stochastically-heated graphite or silicate grains (see Dwek et al. 1997; Figure 2). They are consistent, however, with those produced by PAHs. The addition of PAHs is therefore necessary in order to reproduce the short wavelength diffuse IR emission observed by the COBE.

Calculation of the IR emission requires knowledge of the composition, abundances, and size distribution of the dust particles, and the spectrum and intensity of the ambient radiation field. In the CFD model, all the dust parameters are determined by the fit to the interstellar 
extinction curve, except for the size distribution of the bare silicate and graphite grains. Their size distribution was not specified by Mathis (1996) since it does not affect the extinction as long as the dust grains are Rayleigh particles. For the purpose of our calculations we adopted an MRN power law distribution, $d n(a) / d a \sim a^{-3.5}$ for the bare silicate and graphite grains in the 0.005 to $0.015 \mu \mathrm{m}$ radius interval. The upper limit corresponds to the largest grain radius for which the value of $Q_{e x t} / a$ is still independent of grain size.

Dust in the diffuse high latitude clouds is heated by the local interstellar radiation field (LISRF), and for calculational purposes we adopted the LISRF spectrum and intensity from the studies of Mathis, Mezger, \& Panagia (1983). With the dust model and interstellar radiation field specified, we calculated the emerging IR emission without further adjustments of any model parameters. Our calculations include the effects of the stochastic heating and temperature fluctuations on the IR dust spectrum (see Dwek et al. 1997 for details).

Figure 3 compares the IR emission predicted by the CFD model to the observations. With the addition of PAHs, the model gives a very good fit to the observed intensity at $\lambda \lesssim 140 \mu \mathrm{m}$. PAHs radiate about $25 \%$ of the total diffuse IR emission (Dwek et al. 1997). However, at longer wavelengths the model produces a large excess of emission over that detected by the $C O B E$ satellite. As shown below, the enhanced IR emission is an unavoidable consequence of the UV-optical properties of the CFD particles.

\subsection{The Interstellar Extinction and Dust Albedo}

Figure 4 presents the extinction predicted by the CFD model in the various wavelength regimes. Figure 4 a depicts the UV-optical portion of the curve as a function of $x \equiv 1 / \lambda(\mu m)$. The thin line in the figure shows the extinction presented by Mathis (1996) without PAHs. The model provides a very good fit to the data, deviating by less than $10 \%$ over the given range of $x$. The fit would have been better if, following Mathis (1996), we had modified the optical constants of the silicate grains in the $6-8 \mu \mathrm{m}^{-1}$ region to elliminate an artificial kink in their extinction curve at $\sim 7 \mu \mathrm{m}^{-1}$. The bold line in the figure depicts the total extinction when PAHs are included in the dust model. The calculated extinction is now significantly higher than the observed one, which should not be surprising, since PAHs radiate about $25 \%$ of the total diffuse IR emission. The shape of the PAH extinction is highly uncertain. Here we adopted the extinction properties of the PAHs from the work of Désert et al. (1990), which were chosen to reproduce the general interstellar extinction curve with their dust model. However, regardless of the exact form of their UV-optical cross sections, PAHs (or any other carriers of the UIBs) must be responsible for about $25(1-A) \%$ of the integrated extinction in the $1 / \lambda=1-10 \mu \mathrm{m}^{-1}$ interval, where $A$ is the average albedo at UV-optical wavelength.

Figure $4 \mathrm{~b}$ compares the extinction produced by the CFD model (including PAHs) to the observed infrared extinction (Mathis 1990; Table 1). The model produces higher extinction at 
wavelengths $\gtrsim 10 \mu \mathrm{m}$, but it is nevertheless consistent with the observations considering the fact that uncertainties in the data are at least a factor of two at $\lambda \gtrsim 15 \mu \mathrm{m}$.

Figure 5 depicts the albedo of the CFD model as a function of $x \equiv 1 / \lambda(\mu m)$ (bold line). For comparison, we also plotted the albedo predicted by the MRN model with Draine-Lee optical constants (dotted line). In particular, the visual albedo of the MRN model is about 0.6, consistent with the value of $0.61 \pm 0.07$ suggested by Witt (1989). The albedo of the CFD model is lower, about 0.5 , a fact already pointed out by Mathis (1996). So in spite of the fact that both, the MRN and the CFD, models reproduce the observed interstellar extinction equally well, the CFD model has a lower albedo over the whole UV-optical wavelength range. The composite fluffy dust particles therefore absorb more energy from the LISRF than the bare silicate graphite grains in the MRN model. This energy has to be reradiated at IR wavelengths, producing an excess far-IR emission compared to that produced by an MRN distribution of bare graphite and silicate grains.

\subsection{The Carbon Abundance}

The main motivation for the composite dust model was to solve the interstellar carbon crisis. The original MRN model requires a $\mathrm{C}$ abundance of about 270 atoms to be in the dust (using Draine-Lee optical constants), significantly above the value of $85 \pm 55$ implied from the recent ISM values. Kim \& Martin (1996) optimized the grain size distribution, and produced a similar C abundance of $270 \pm 50$ atoms in carbon dust. The CFD model of Mathis takes therefore a large step towards easing the carbon crisis, requiring only $\sim 160 \mathrm{C}$-atoms to be in the dust phase. However, the model ignores the amount of carbon locked up in PAHs which was recently estimated from the $C O B E$ data to be $70 \pm 20 \mathrm{C}$-atoms (Dwek et al. 1997). This PAH abundance is higher by about a factor of two compared to previous estimates (e.g. Désert et al. 1990; Siebenmorgen \& Krügel 1992). However, recent observations of the $6.05 \mu \mathrm{m}$ absorption feature in the ISM with the Infrared Space Observatory (ISO; Schutte et al. 1996) suggest a similarly large value. Solid $\mathrm{H}_{2} \mathrm{O}$ provides the bulk of the absorption in the $6.05 \mu \mathrm{m}$ band. However, an excess absorption in the red wing of the feature is due to the $\mathrm{C}-\mathrm{C}$ stretching mode, and the authors estimate that about $20 \%$ of the solar carbon abundance (they adopt $(\mathrm{C} / \mathrm{H})_{\odot}=10^{-4}$ ) must be in this bond to produce the observed absorption. If this bond is in PAHs, then these observations confirm the PAH abundance estimates of Dwek et al. (1997). If we add PAHs to the composite dust model we get that it will require a total $\mathrm{C}$ abundance of $225 \pm 20$ atoms (55 in small graphite grains, 105 as amorphous carbon in composite grains, and 70 in PAHs). This carbon abundance is smaller than any other dust model [see Kim \& Martin (1996) or Snow \& Witt (1995) for a summary of the carbon abundance requirements of the various dust models], but still significantly larger that the maximum available for the dust in the ISM. 


\subsection{PAHs as Carriers of the $2200 \AA$ extinction feature?}

One way to ease the carbon abundance constraint is to assume that PAHs are the carriers of the $2200 \AA$ extinction bump as well as the UIBs. This assumption will reduce the $\mathrm{C}$ abundance by 55 atoms, the amount locked up in graphite grains. There are several arguments in favor of such an identification: (1) Laboratory measurements show that small PAHs have an enhanced UV absorptivity around $2200 \AA$ (Joblin, Léger, \& Martin 1992). Peak cross sections are about $810^{-18} \mathrm{~cm}^{2}$ per C-atom. For an opacity of $\tau_{2200} / N_{H}=5.2710^{-22} \mathrm{~cm}^{-2}$, and a Drude profile for the emission bump (e.g. Draine 1989), the required $\mathrm{C}$ abundance in PAHs is $\sim 65$ atoms, comparable with the amount required to produce the UIB emission features; (2) extinction measurements along several lines of sight through the Chamaeleon cloud (Boulanger, Prévot, \& Gry 1994) show a corelation between the strength of the $2200 \AA$ bump and the magnitude of the IRAS $12 \mu \mathrm{m}$ emission. Since most of the $12 \mu \mathrm{m}$ emission originates from PAHs, the correlation suggests that PAHs can be significant contributors to the UV extinction bump.

The problem with PAHs is that the peak wavelength of the UV emission bump and its profile seems to vary with PAH composition. Furthermore, the PAH cross sections exhibit a feature around $3000 \AA$ (Joblin et al. 1992) that is not observed in the ISM. It yet remains to be seen if a

suitable mixture of PAHs will match the interstellar $2200 \AA$ extinction profile and smooth out the unobserved UV feature.

Even if PAHs can be substituted for graphite, the elimination of these particles will create an additional problem for the CFD model. The very small graphite particles are not only responsible for the $2200 \AA$ extinction, but also contribute to the observed 25 - $60 \mu \mathrm{m}$ excess emission, since they are stochastically heated by the LISRF. A population of very small particles will therefore be needed to account for the observed mid-IR emission, which is currently absent in the size distribution of the composite grains.

\section{SUMMARY}

In this paper we showed that composite interstellar dust particles consisting of amorphous carbon and silicate dust with vacuum comprising $45 \%$ of their volume cannot be a major interstellar dust component in the diffuse ISM. Heated by the local interstellar radiation field, these particles produce a significantly higher IR emission than observed by the COBE/DIRBE and FIRAS instruments for the diffuse high latitude emission. The excess IR emission is the result of the increased absorptivity of the composite fluffy dust particles at UV-optical wavelengths. The robustness of this conclusion depends on the validity of the calculations of the optical properties of composite dust particles. A different rule for producing the average optical properties of the composite particles may produce an IR emissivity that is consistent with the COBE data, but it will certainly affect the UV-optical extinction as well, requiring a whole new assesment of the composite dust model. 
The neglect of the composite fluffy dust model (or any other dust model) to include the carriers of the unidentified IR emission bands (UIBs) will result in an underestimate of the carbon abundance by $\sim 20 \%$ of its solar value, and will introduce a $f(1-A) \%$ uncertainty in the UV-optical part of the interstellar extinction curve, where $A \approx 0.6$ is the average UV-optical albedo of the dust, and $f \approx 0.25$ is the fraction of the total IR radiation emitted by these particles. Since the shape of the UV-optical absorptivity of the coal or PAHs that are responsible for the UIBs is unknown, it introduces a major uncertainty in any interstellar extinction model.

We point out that the inferred abundance of the UIB carriers is inversely proportional to their wavelength-averaged UV-optical absorption cross section. Lowering the amount of carbon in these particles will require an increase in their UV-optical extinction, and conversely, minimizing their effects on the extinction will require a high $\mathrm{C}$ abundance to be tied up in these carriers. To summarize, the composite fluffy dust model does not reproduce the UV-optical extinction to better than about $12 \%$, and with the addition of UIB carriers it uses up twice the amount of the carbon believed to be available in the ISM for the dust.

Since the composite dust model falls short in solving the carbon crisis it is worth examining other possible solutions to this crisis. The major assumption leading to this crisis is that the dust abundance should reflects that of the current local ISM. This assumption requires that the ISM gas and dust have identical spatial and temporal histories. However, there is evidence to suggest that this may not be the case: (a) observations of a large sample of stars in the solar neighborhood (e.g. Edvardsson et al. 1993) shows an intrinsic scatter in their observed abundances as a function of time and metallicity; (b) the presence of isotopic anomalies in meteorites suggests that the solar neighborhood may have been polluted by the nucleosynthetic products of a neigboring stars and supernovae (e.g. Zinner 1996); (c) additional inhomogeneities may be introduced by dynamical fractionation between gas and dust in star formation processes. Numerical simulations of protostellar collapses (Ciolek \& Mouschovias 1996) show that ambipolar diffusion can "strain" the infalling material, leaving the grains behind and reducing their abundance in the collapsed core which is destined to become a star.

We conclude that inhomogeneous chemical mixing and fractionation effects in the ISM may be responsible for the differences in metallicities between the sun and the local ISM, and perhaps between that inferred from the B stars and the extinction as well.

I acknowledge the Institut d'Astrophysique Spatiale in Orsay, and the Institut d'Astrophysique de Paris for providing a stimulating atmosphere and environment for the duration of my research. During my work I benefitted from many stimulating discussions with Francois Boulanger, Xavier Désert, Ant Jones, Alain Léger, Alain Omont, Renauld Papoular, Jean-Loup Puget, and Bill Reach. Special thanks are due to John Mathis who provided stimulating discussions, some intermediate results of his model which were used to check current calculations, and comments on a preliminary version of the manuscript. Comments by an anonymous referee led to further improvement in the manuscript. Finally, I acknowledge the NASA/GSFC Research and Study 
Fellowship Program for providing financial assistance during my stay at these institutes.

\section{REFERENCES}

Arendt, R. G. et al. 1997, in preparation

Bernard, J. -P., Boulanger, F., Désert, F.,-X., Giard, M., Helou, G.,\& Puget, J. -L. 1994, A\&A, 291, L5

Bohren, C. F., \& Huffman, D. R. 1983, Absorption and Scattering of Light by Small Particles (Wiley: New York)

Boulanger, F., Prévot, M. L., \& Gry, C. 1994, A\&A, 284, 956

Cardelli, J. A., Clayton, G. C., \& Mathis, J. S. 1989, ApJ, 245, 345

Cardelli, J. A., Meyer, D. M., Jura, M., \& Savage, B. D. 1996, ApJ, 467, 334

Ciolek, G. E., \& Mouschovias, T. Ch. 1996, ApJ,468, 749

Désert, F. -X., Boulanger, F., \& Puget, J. L.1990, A\&A, 237, 215

Draine, B. T., \& Lee, H. M. 1984, ApJ, 285, 89 bibitem[Draine \& Anderson 1985]da85 Draine, B. T., \& Anderson, N. 1985, ApJ, 292, 494

Draine, B. T. 1985, ApJS, 57, 587

Draine, B. T. 1989, in IAU Symp. No. 135, Interstellar Dust, eds. L. J. Allamandola \& A. G. G. M. Tielens (Boston: Kluwer), p. 313

Dwek, E. et al. 1997, ApJ, 475, 000

Edvardsson, B., Andersen, J., Gustafsson, B., Lambert, D. L., Nissen, P. E., \& Tomkin, J. 1993, A\&A, 275, 101

Grevesse, N., Noels, A., Sauval, A. J. 1996, in Cosmic Abundances, ASP Conference Series, eds. S. S. Holts, \& G. Sonneborn (San Francisco: ASPCS), p. 117

Joblin, C., Léger, A., \& Martin, P. 1992, ApJ, 393, L79

Kim, S-H, Martin, P. G. 1996, ApJ, 462, 296

Mathis, J. S., Rumpl, W., \& Nordsieck, K. H. 1977, ApJ, 217, 425, MRN

Mathis, J. S., Mezger, P. G., \& Panagia, N. 1983, A\&A, 128, 212

Mathis, J. S., Whiffen, G. 1989, ApJ, 341, 808 
Mathis, J. S. 1990, ARA\&A, 28, 37

Mathis, J. S. 1996, ApJ, 472, 643

Rouleau, F., \& Martin, P. G. 1991, ApJ, 377, 526

Schutte, W. A. 1996, A\&A, 315, L333

Siebenmorgen, R., \& Krügel, E. 1992,A\&A, 259, 614

Snow, T. P., \& Witt, A. N. 1995, Science, 270, 1455

Weiland, J. L., Blitz, L., Dwek, E., Hauser, M. G., Magnani, L.,\& Rickard, L. J. 1986, ApJ, 306, L101

Weiland, J. L., Arendt, R. G., \& Sodroski, T. J. 1996, in AIP Conf. Proc. No. 348, Unveiling the Cosmic Infrared Background, ed. E. Dwek (New York: AIP), p. 74

Witt, A. N. 1989, in Interstellar Dust, eds. L. J. Allamandola \& A. G. G. M. Tielens (Dordrecht: Kluwer), p. 87

Zinner, E. 1996, in Cosmic Abundances, ASP Conference Series, eds. S. S. Holts, \& G. Sonneborn (San Francisco: ASPCS), p. 147 
Fig. 1.- (a) The values of the real part of the dielectric constant of amorphous carbon of type Be (dashed line) and silicate (light solid line) as a function of wavelength. The dotted line at $\epsilon$ (real) $=1.0$ represents the vacuum. The bold line is the value of $\epsilon$ (real) of the composite fluffy grain obtained from equation (1). (b) The same as (a) for the imaginary part of the dielectric constant.

Fig. 2.- The value of $Q_{e x t} / \mathrm{a}$ in the Rayleigh approximation (see eq. (3) in the text) is plotted as a function of wavelength for Be-type amorphous carbon of type Be (dashed line), silicate (light solid line), and the composite grains (bold line). Averaging the optical constants of the constituent grains yields an enhanced emissivity for the composite particle.

Fig. 3.- Comparison of the infrared spectrum predicted by the composite dust model (bold line) to the average dust spectrum in the diffuse ISM observed by the COBE satellite (DIRBE data are represented by diamonds, FIRAS data by the thin solid line). PAHs have been added to the model to account for the 3.5 to $12 \mu \mathrm{m}$ DIRBE emission. Also shown in the figure is the contribution of PAHs (dotted line), graphite (dashed line), silicates (dashed-dotted line), and the composite particles (solid line).

Fig. 4.- The extinction of the composite dust model is compared to the observed average interstellar extinction curve. (a) Comparison to the UV-optical extinction given by Cardelli, Mathis, \& Clayton (1989). The addition of PAHs to the composite dust model produces an excess in the extinction (bold line) over the observations. The thin solid line is the total extinction calculated by Mathis (1996) without PAHs. Also shown in the figure are the contributions of graphite (dashed line), silicates (dotted line), and the composite grains (dashed dotted line) to the total extinction. (b) Comparison of the calculated extinction (bold line) to the infrared extinction (Mathis 1990; diamonds). The excess extinction predicetd by the composite dust model is within the uncertainty of the observations.

Fig. 5.- The albedo of the composite fluffy dust (CFD) model (solid line) is plotted versus inverse wavelength. Also shown is the albedo of the MRN model, calculated with Draine-Lee optical constants. The low albedo (high absorptivity) of the CFD model is the cause of the excess far-IR emission depicted in Figure 3. 


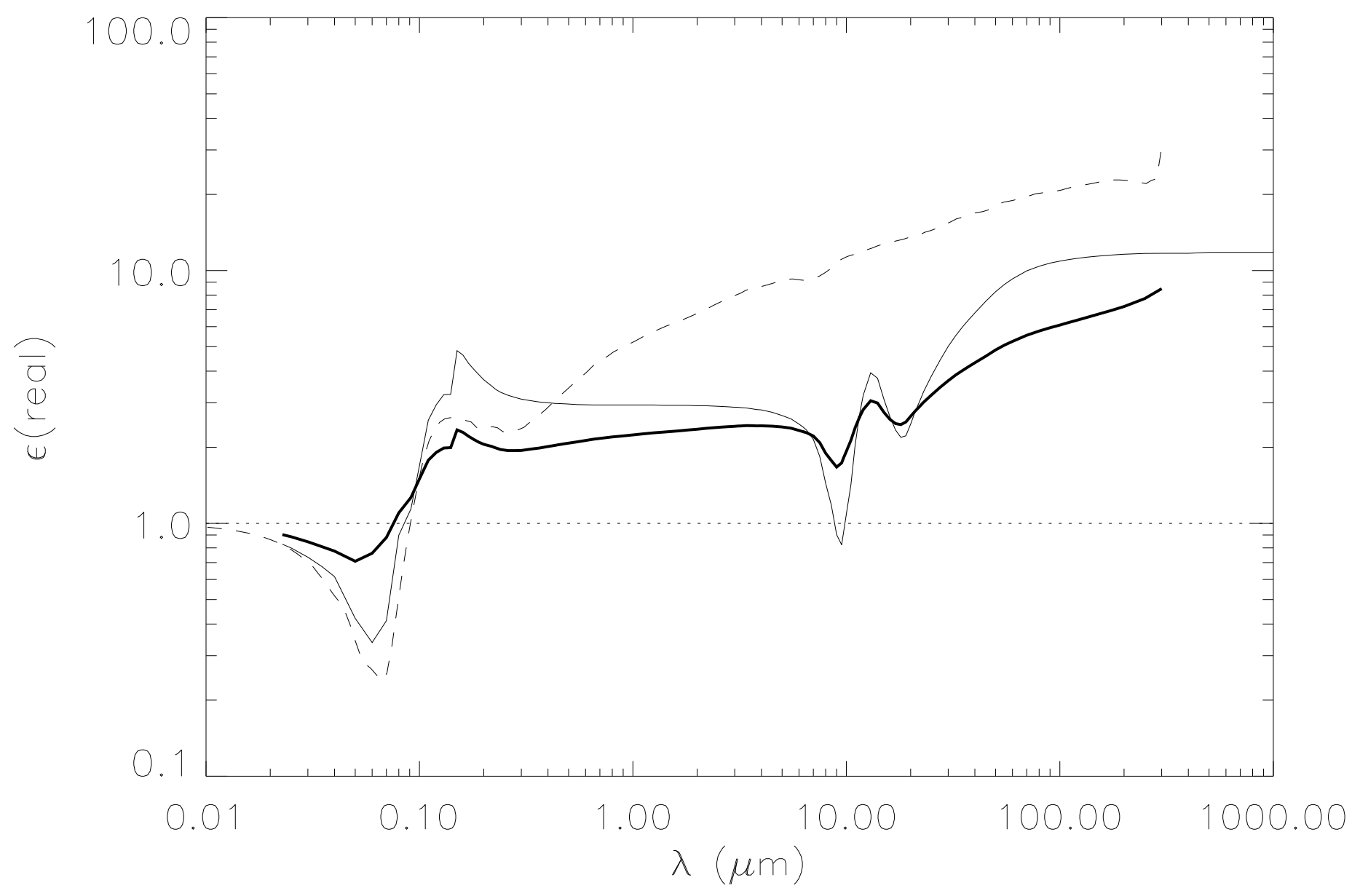




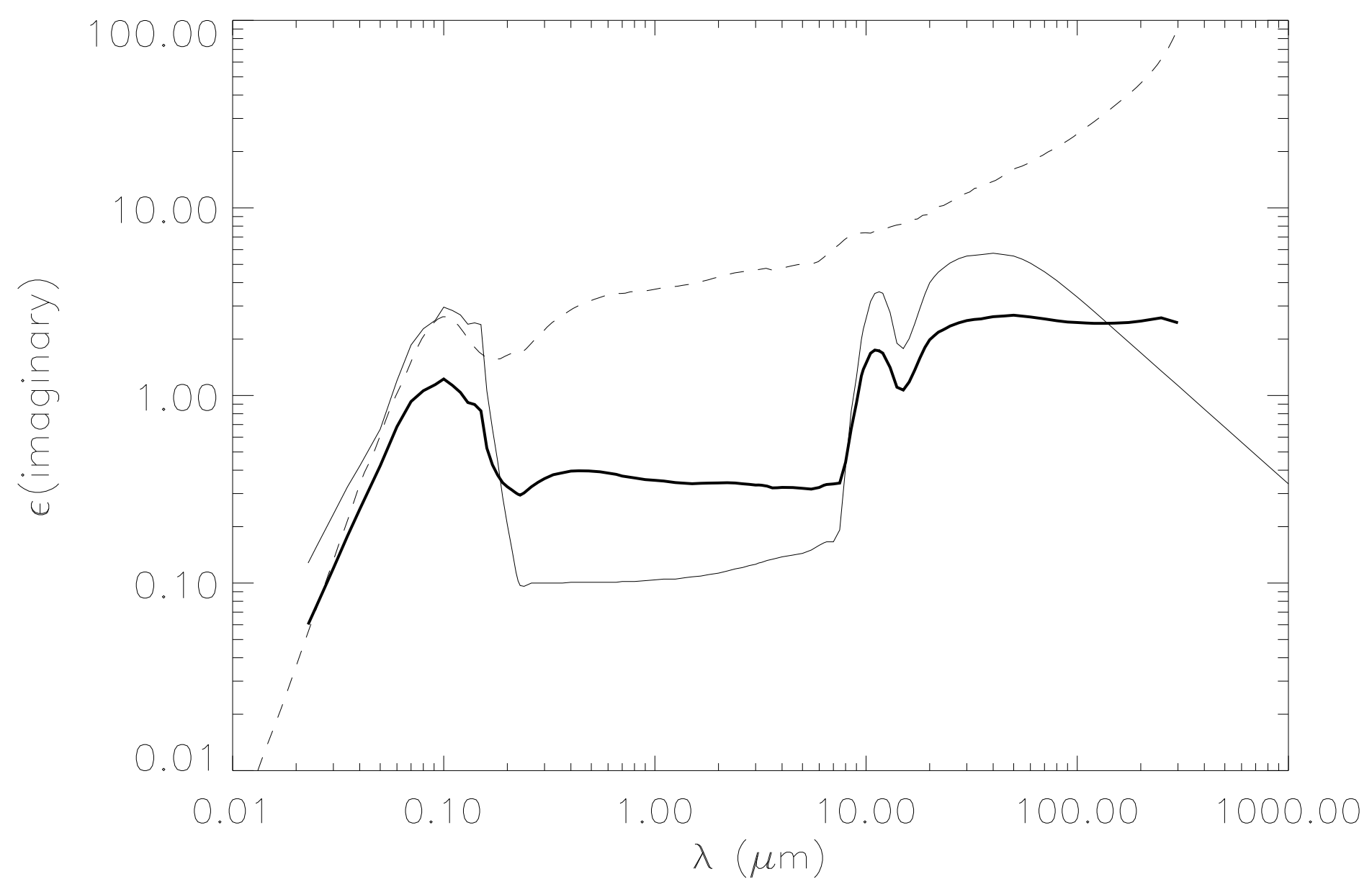




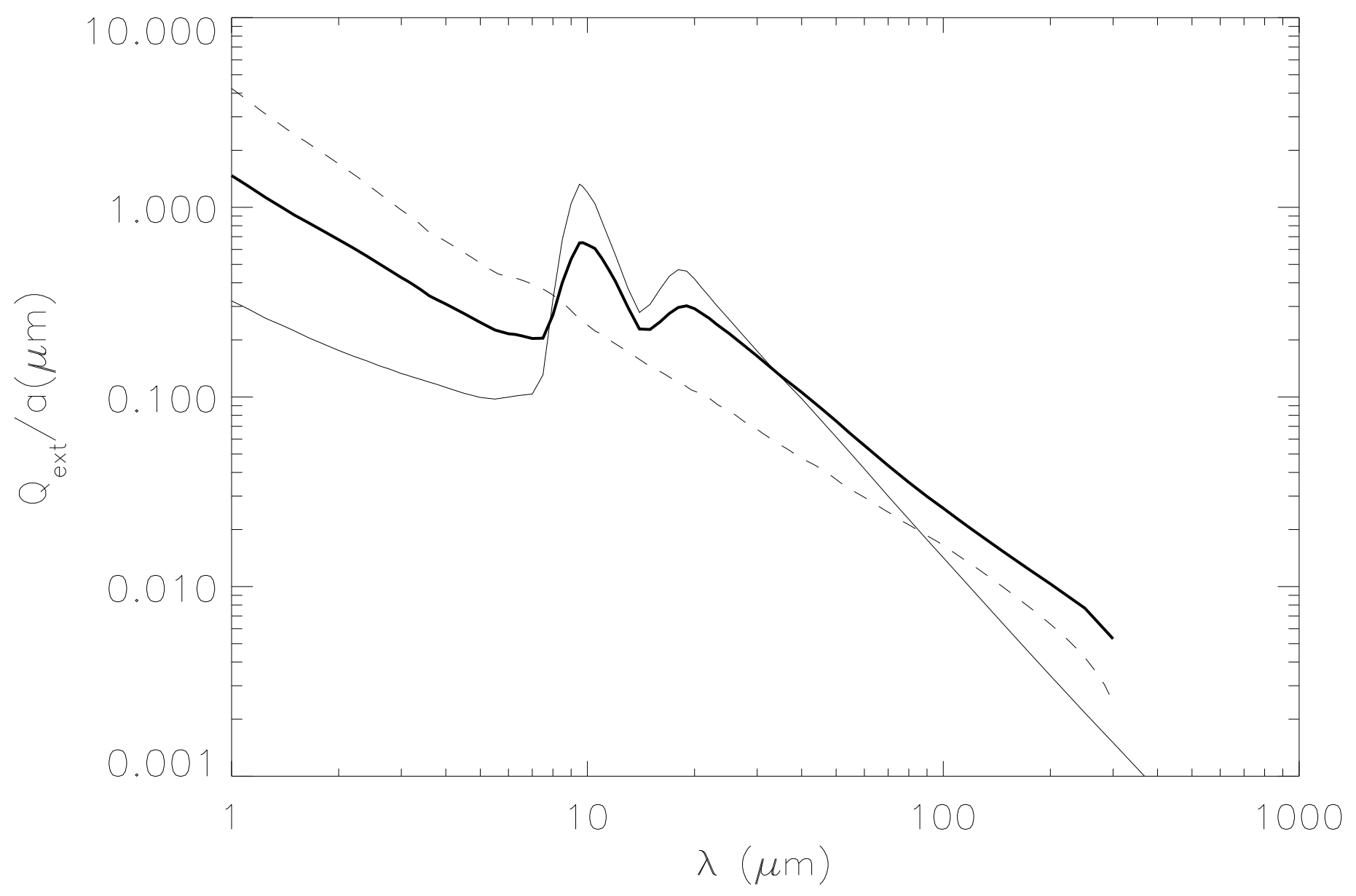




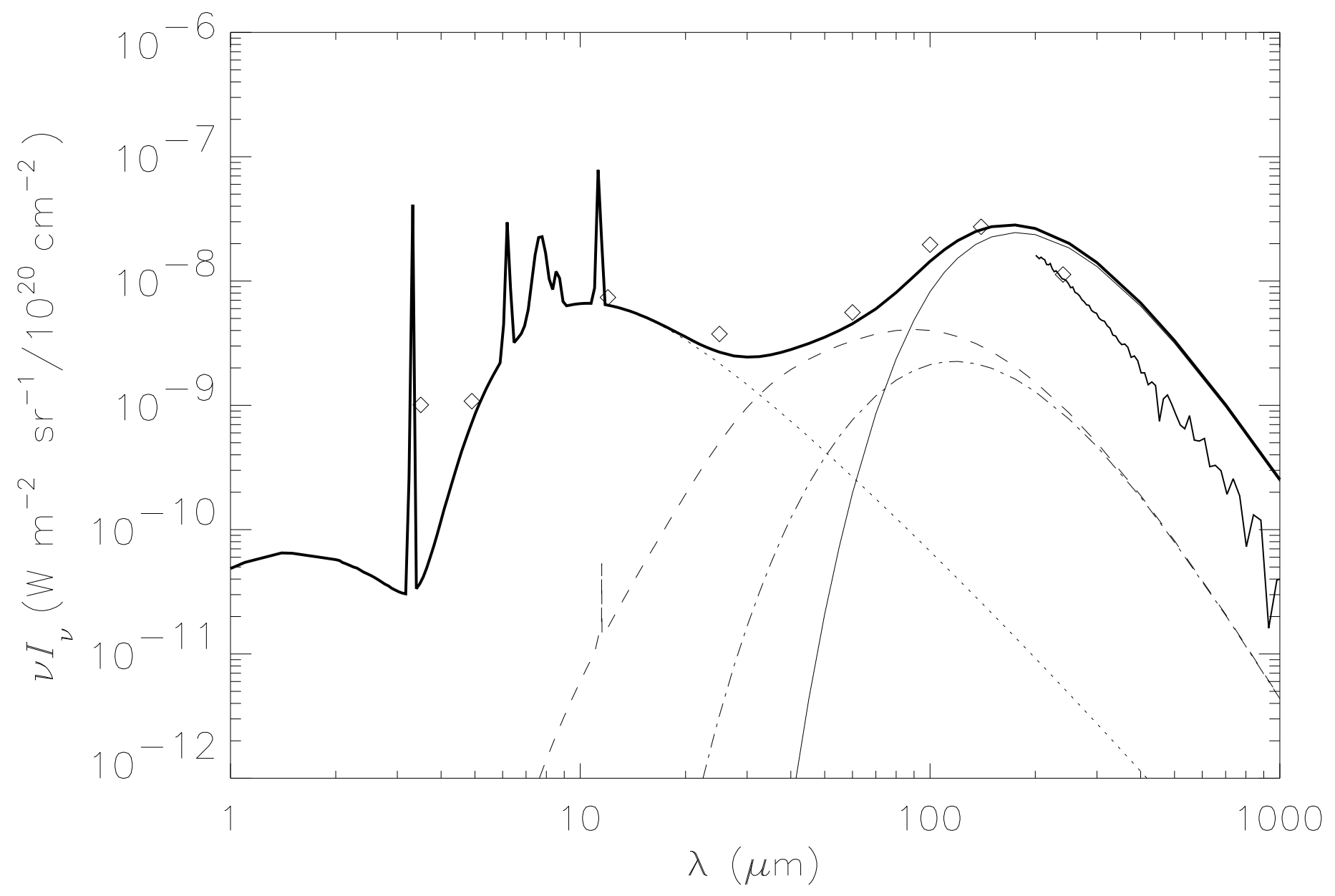




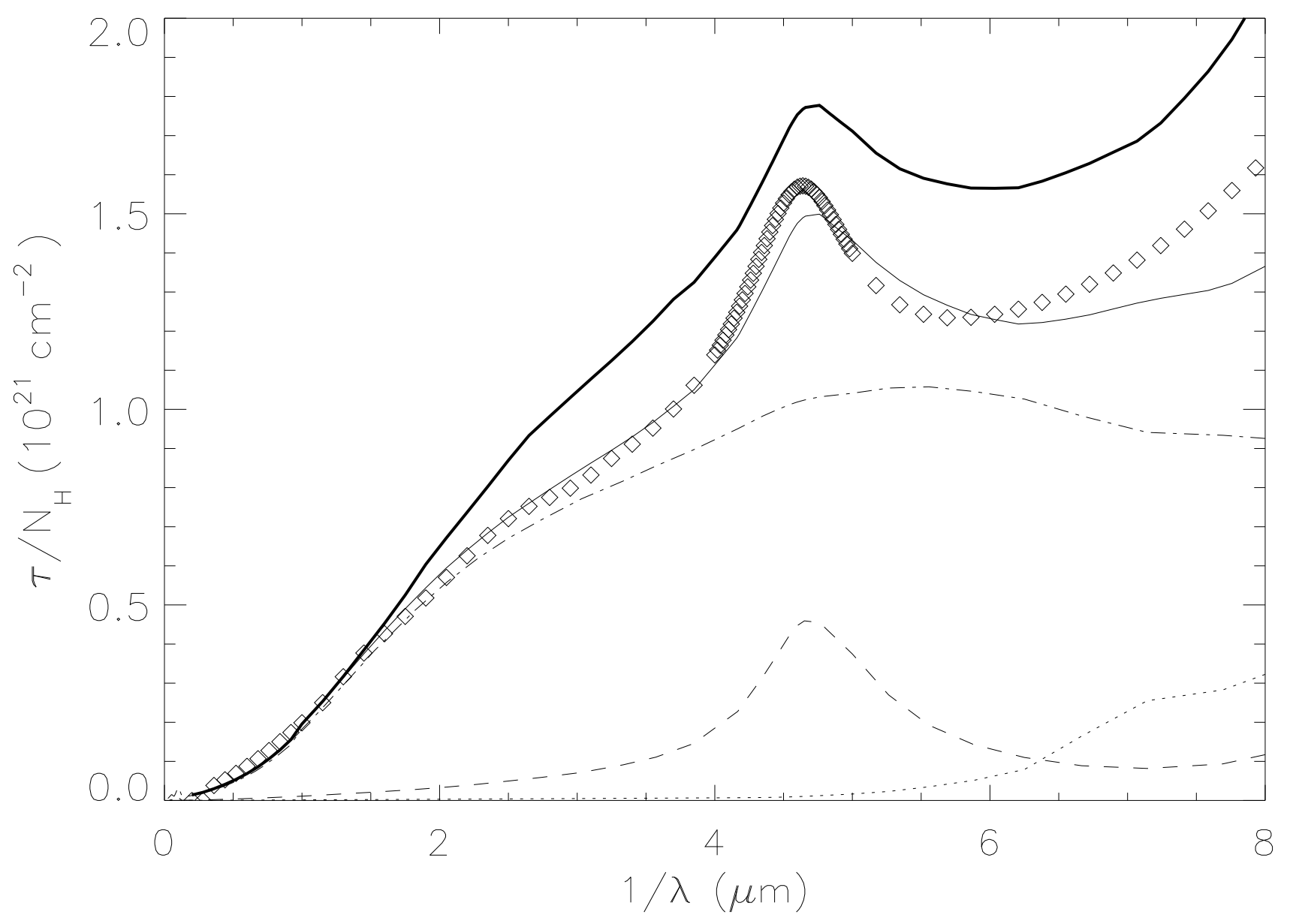




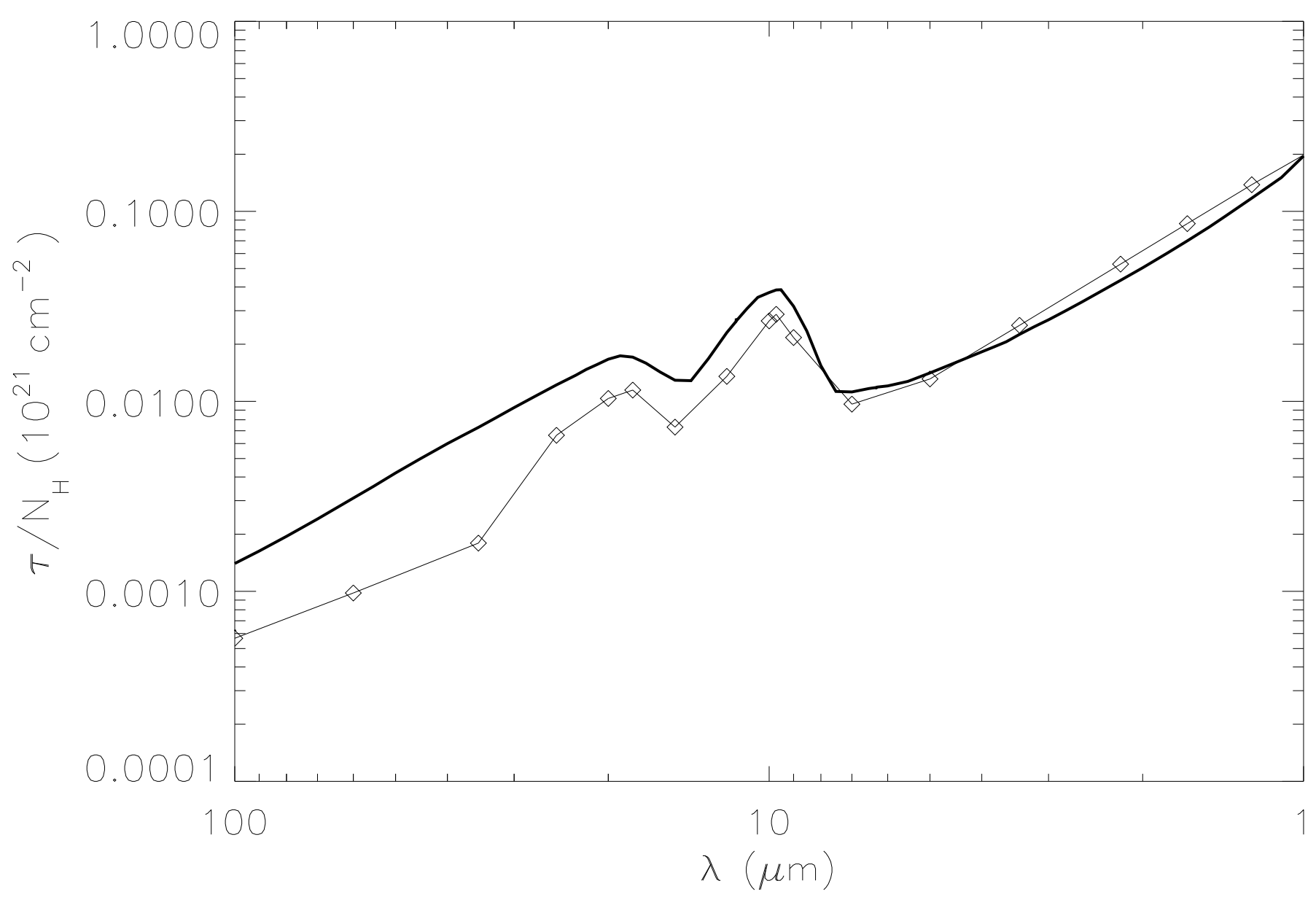




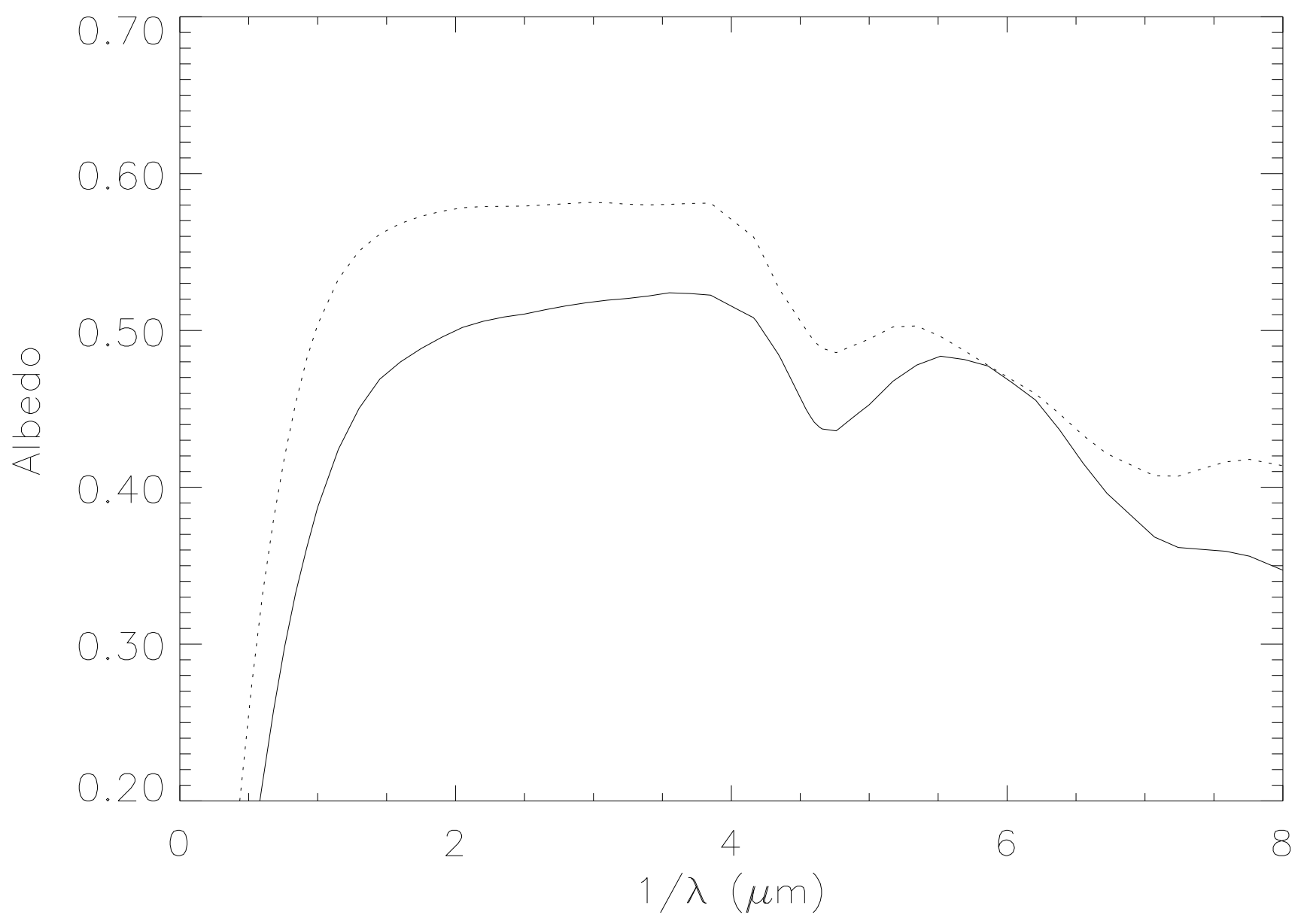

